\title{
Collective bubble dynamics near a surface in a weak acoustic standing wave field
}

\author{
Xiaoyu Xi ${ }^{1}$, Frederic Cegla ${ }^{1}$, Robert Mettin ${ }^{2}$, Frank Holsteyns ${ }^{3}$, and Alexander Lippert ${ }^{3}$ \\ ${ }^{1}$ Department of Mechanical Engineering, Imperial College London, London SW7 2AZ, UK \\ ${ }^{2}$ Christian Doppler Laboratory for Cavitation and Micro-Erosion, Drittes Physikalisches \\ Institut, Georg-August-Universität Göttingen, Friedrich-Hund-Platz 1, 37077 Göttingen, \\ Germany \\ ${ }^{3}$ Lam Research AG, SEZ-Strasse 1, 9050 Villach, Austria
}




\begin{abstract}
The transport of bubbles to a neighboring surface is very important in surface chemistry, bioengineering, and ultrasonic cleaning etc. This paper proposes a multi-bubble transport method by using an acoustic standing wave field and establishes a model which explains the multi-bubble translation by expressing the balance between Bjerknes forces and hydrodynamic forces on a bubble in a liquid medium. An uniform one-dimensional acoustic standing wave field was created by a multi-layered resonator which was designed based on a one-dimensional equivalent network model. A pair of modified Keller-Miksis equation and translation equations, which take into account the influence from boundary surfaces and neighboring bubbles, were used to simulate the bubble translations. The bubble translations were observed by a high speed camera system. Results indicated that the bubble translations were mainly influenced by the acoustic wave field, the boundary, neighboring bubbles, and buoyancy force from the surrounding liquid. The primary Bjerknes force generated by the sound field dominated the bubble translations at the beginning when the bubbles were far away from the boundary. A surge of attractive secondary Bjerknes force from the boundary was seen when the bubbles were approaching the boundary. The attraction force outweighed the primary Bjerknes force within a short distance of the surface and resulted in a faster bubble motion. Besides the forces generated from the acoustic field and the boundary, neighboring bubbles also exerted secondary Bjerknes forces on a target bubble and influenced its translation. Moreover, to optimize the bubble translation in a multi-bubble environment, a parametric study was carried out to investigate the influence of varied bubble size and acoustic pressure amplitudes on the bubble translation. It was found that increasing the size of a bubble can hardly alter its trajectory but only force it to move at a faster speed. An increase of pressure amplitude can also accelerate the bubble motion and enhance the bubble-bubble interaction. The secondary Bjerknes force between two bubbles can switch from an attractive one when they oscillate in phase, to a repulsive one when the bubble oscillations are out of phase. These findings provide an insight into the multi-bubble translation near a surface and can be applied to future bubble motion control studies, especially in drug delivery, sonoporation, and ultrasonic cleaning.
\end{abstract}




\section{Introduction}

It has been recognized that acoustically-driven bubbles can trigger substantial physical and chemical effects on a neighboring surface $[1,2]$. Extensive experimental investigations have been carried out over the past few decades to understand the interactions between cavitation bubbles and surfaces of different properties, from a solid plane surface [3] to an elastic membrane [4]. It was found that the liquid jet which is formed during the collapse of a bubble near a boundary is responsible for a range of bubble induced physical and chemical reactions and such liquid motion is strong enough to erode a solid surface [5] or clean contaminated wafers [6-8]. Numerical calculations of cavitation bubbles near a boundary are also found in the literature [9-11]. Lauterborn and Kurz recently published a comprehensive review of this topic [12].

Despite the successful investigations of interactions between cavitation bubbles and a surface, only recently, the study of the acoustic response of moderately oscillating bubbles near a surface started to attract more and more attention due to its importance in understanding sonoporation and drug delivery. At a low pressure amplitude, a bubble experiences a moderate oscillation and consequently induces a flow circulation around it. The induced fluid flow is assumed to be capable of gently disturbing the fluid flow near a surface or opening breaches on cell membranes [13]. Marmottant et al [14-16] explored the micro flow circulation generated by a bubble attaching to a solid surface. The change in micro-bubble dynamics near a solid wall was revealed by Garbin et al [17]. Meanwhile, Vos et al $[18,19]$ experimentally investigated the deformed radial oscillation of a micro-bubble on a cellulose wall. Besides these experimental studies, a series of numerical analyses were carried out by Doinikov [20-22] to explain the acoustic response of ultrasound contrast agents (UCAs) near 
a boundary, especially the shear stress generated by an UCA on a surface which is believed to be the mechanism of bubble induced sonoporation or cell lysis. However, direct correlation between bubble oscillation and the consequent biological reactions has not yet been quantified. Moreover, in the mentioned studies, cavitation bubbles were normally generated near a target surface by a focused laser beam. Such approach, however, is difficult to accurately control the force generated by the target bubbles. As an alternative solution, oscillating bubbles are required to be transported to a surface within a short time. Therefore, there is a need to explore the means to effectively transport a large amount of bubbles to an appointed location on a designed trajectory and to manipulate the bubble oscillation in a controlled manner.

The motion of a bubble in a bulk liquid medium is controlled by acoustic and hydrodynamic forces simultaneously. A gas bubble driven below its resonance frequency in a weak standing wave field moves towards the pressure anti-node, while a bubble driven above its resonance frequency moves towards the pressure node instead. This effect is attributed to the primary Bjerknes force on a bubble and has been studied extensively by several authors [23-27]. Besides the primary Bjerknes force generated by an imposed acoustic field, the translation of a bubble can also be influenced by boundary conditions or neighboring oscillating bubbles, which exert secondary Bjerknes forces on the target bubble. The secondary Bjerknes force takes effect between two nearby bubbles since the force is inversely proportional to the square of the separation distance between two bubbles [28, 29]. A bubble can exert either an attractive or a repulsive secondary Bjerknes force on a neighboring one, depending on the driving frequency and the bubble sizes [30]. By taking the bubble-bubble interaction into account, the formation of a bubble cluster in an acoustic standing wave 
resonator was successfully simulated by Mettin et al [31] and Parlitz et al [32]. Doinikov [33], however, later pointed out that a missing term in Mettin and Parlitz's works may make their model inadequate in other applications, and therefore proposed a more comprehensive model by using a Lagrangian formalism. Apart from these studies, however, little is known of the influence of secondary Bjerknes forces on the translation of a bubble near a surface in a multi-bubble environment. It was the aim of the present study to develop an experimental configuration to investigate the effects of different acoustic and hydrodynamic forces on the bubble translation near a boundary.

Recently, the authors reported a multi-layered resonator for controlling single bubble translation near a surface in an acoustic standing wave field [34]. The resonator can create an uniform one-dimensional standing wave field in a liquid medium. The acoustic characteristics of such a structure (impedance and pressure distribution) were successfully predicted by a one-dimensional equivalent network model (1D model) $[35,36]$. In this paper, the translation of bubbles in a more general multi-bubble environment is presented and the dynamics of bubbles in a weak acoustic standing wave field are discussed in more detail.

This paper is organized as follows. In section 2, details of the experimental setup are described. Section 3 provides the theoretical background of the bubble translation in a multibubble environment. In section 4, experimental results obtained from a high speed camera are shown and are explained by the bubble translation model in section 5 . Conclusions are drawn in section 6 . 


\section{Experimental configuration}

It is well-known that a bubble can move in an acoustic standing wave field either towards a pressure node or a pressure anti-node, depending on the bubble size and the driving frequency. In this study, the standing wave was created by a multi-layered resonator and the motion of bubbles, which were generated by an electrolysis method, was recorded by a high speed camera system.

The main parts of the resonator are a liquid (deionized water) cube held in a brass block (Length $*$ Width $*$ Thickness $=10 \mathrm{~mm}$ by $10 \mathrm{~mm}$ by $8 \mathrm{~mm}$ ) and a round transducer with diameter of $10 \mathrm{~mm}$ (Fig. 1). The origin of the coordinate system $(x=0 \mathrm{~mm}, y=0 \mathrm{~mm}$, $z=0 \mathrm{~mm}$ ) was set at the center of the transducer-liquid interface. To operate the following optical observations in an optical transparent environment, the cross section of the water layer was chosen as a square shape $(10 \mathrm{~mm}$ by $10 \mathrm{~mm})$ and two glass windows were fit on both sides of the liquid medium. As the aim of the present work is to investigate the bubble behavior near a surface, a round borosilicate glass plate (glass 1) of $0.1 \mathrm{~mm}$ thickness (VWR, UK) was placed at $x=4 \mathrm{~mm}$ as the target surface and another glass plate, glass 2 at $x=8$ $\mathrm{mm}$, was used to confine the liquid within the structure.

The transducer was fabricated out of a lead zirconate titanate (PZT) disk (PCM 51, EP Electronic Components Ltd, UK), a backing brass bar, a front brass bar with thickness of 4 $\mathrm{mm}, 13 \mathrm{~mm}$ and $15 \mathrm{~mm}$ respectively.

An electrolysis method was used to generate bubbles of radii ranging from 10 to $50 \mu \mathrm{m}$. Two wires (tin-coated copper) were connected to a DC power supply (TNG 35, Voltcraft, Germany) and the electrical potential was set to $5 \mathrm{~V}$. The free ends of the wires were placed at $\mathrm{x}=5 \mathrm{~mm}$ as shown in Fig. 1 . 


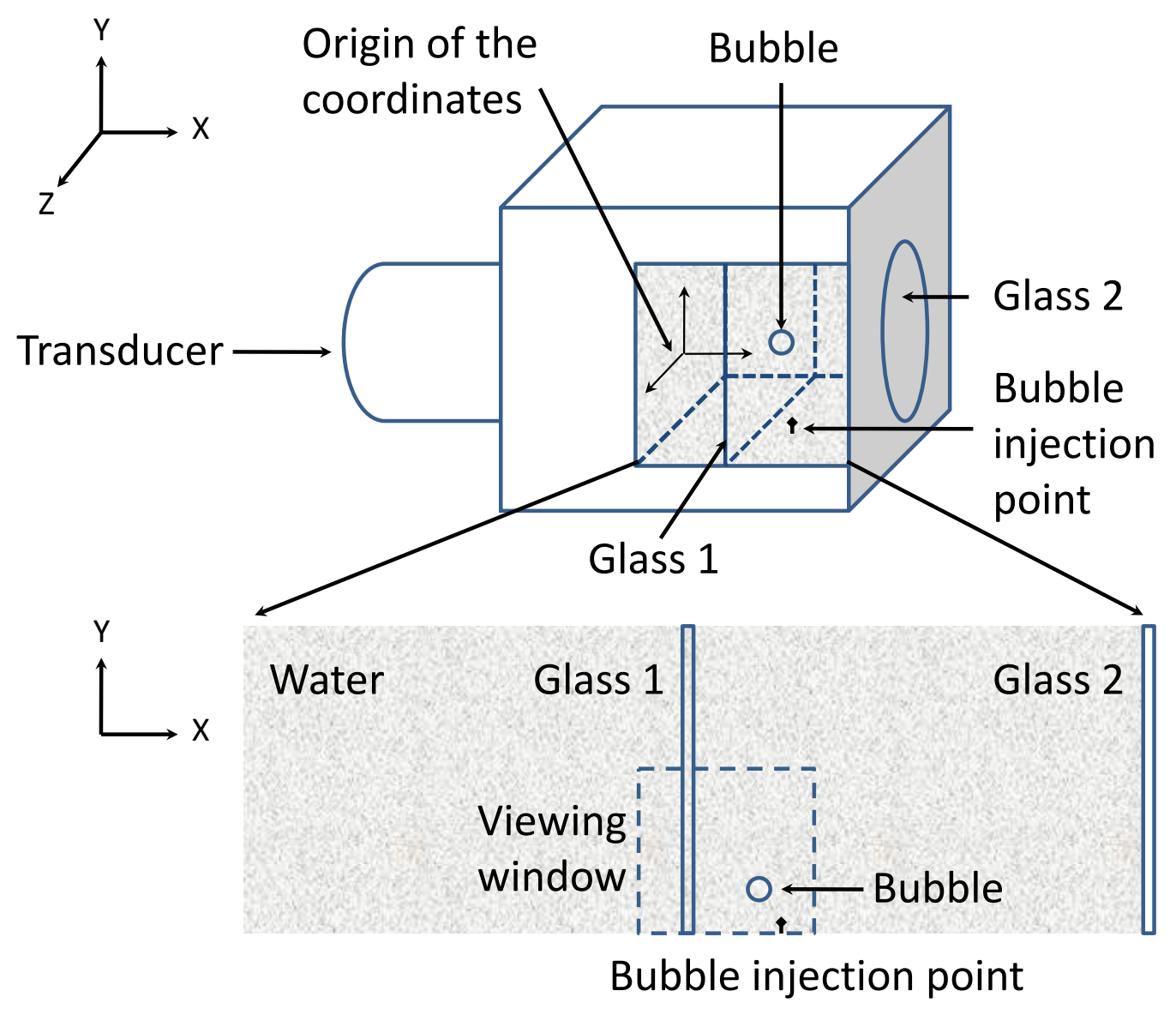

Figure 1: A schematic diagram of the multi-layered resonator for multi-bubble transport.

The bubble motion was recorded by a high speed camera (FastCam SA5, Photron, USA) at a frame rate of 100,000 frames/second. A viewing window with size of $3 \mathrm{~mm}$ by $1.8 \mathrm{~mm}$ was chosen to cover the cross section of glass $1(x=4 \mathrm{~mm})$ and the bubble injection point $(x=5 \mathrm{~mm})$ at the same time. The recorded bubble translation as a function of time was analyzed by an object tracking algorithm written in Matlab (Mathworks Inc, USA). The dimensions of objects in a video were calibrated with a standard $300 \mu \mathrm{m}$ width stick. 


\section{Bubble translation model}

When a bubble moves within the resonator (Fig. 1), its motion is controlled by acoustic and hydrodynamic forces simultaneously. A schematic diagram of the multi-bubble environment is as shown in Fig. 2 and Fig. 3 demonstrates the relationship between different external forces on a target bubble (bubble 1 ). The translation of bubble 1 is mainly influenced by the imposed acoustic field, the boundary surfaces (glass 1 and 2), a nearby bubble 2 , and the buoyancy force from the surrounding liquid.

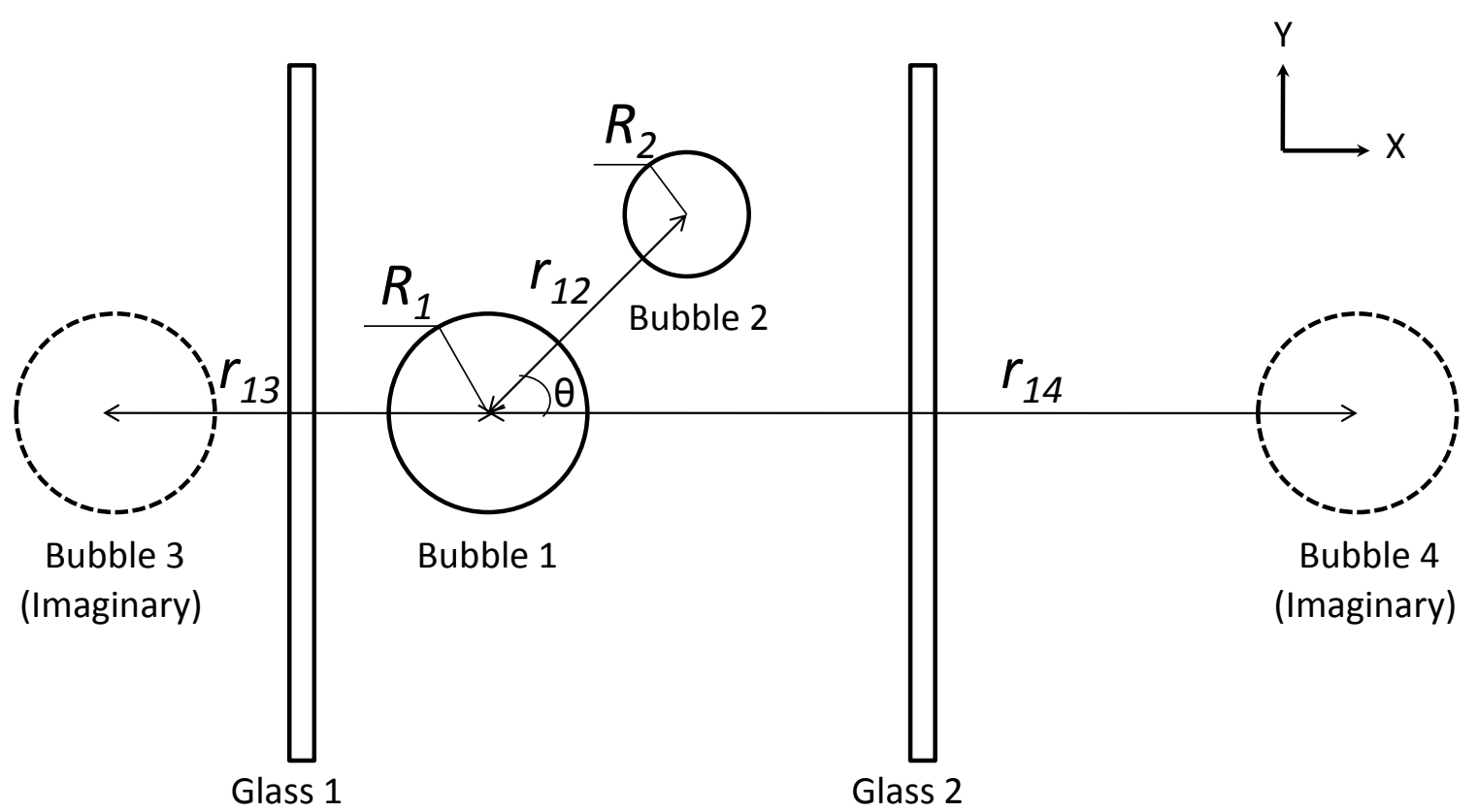

Figure 2: The position relationship between a bubble and its imaginary counterparts and a neighboring bubble in a sound field.

In the multi-bubble environment, the bubble translation is influenced by the primary Bjerknes force $\left(F_{p}\right)$ generated by the acoustic field in the $\mathrm{x}$ axis [37].

$$
F_{p}=-\frac{4 \pi}{3} R_{n}^{3} k P_{a} \sin (\omega t) \cos \left(k d_{n}\right)
$$


where $R_{n}$ is the radius of the $n$th bubble, $P_{a}$ is the pressure amplitude, $\omega$ is the angular frequency, $t$ is time and $k$ is the wave number. $d_{n}$ is the distance between the center of the $n$th bubble and a pressure anti-node along the $\mathrm{x}$ axis in Fig. 2 .

Glass 1 and 2 exert attractive secondary Bjerknes forces on bubble 1. Mathematically, the attractive forces can be represented by introducing imaginary bubbles on the opposite side of the surfaces. In Fig. 2, for example, bubble 1 and an imaginary bubble (bubble 3), which oscillates in phase with its counterpart, are placed equally on each side of glass 1. Moreover, the nearby bubble 2 can exert either an attractive secondary Bjerknes force on bubble 1 when they oscillate in phase, or a repulsive force when their oscillations are out of phase at the driving frequency.

For a pair of bubbles, if the bubble shapes are assumed to remain spherical for all time with the radii $R_{1}$ and $R_{2}$ respectively, the respective pressure, for example, generated from bubble 2 on bubble 1 , is given by [30]:

$$
p=\frac{\rho}{r_{12}} \frac{\mathrm{d}}{\mathrm{d} t} R_{2}^{2} \dot{R}_{2}
$$

where $r_{12}$ is the separation distance between the two bubbles. $\rho$ is the liquid density. The over dot denotes the time derivative.

The secondary Bjerknes force between the bubbles is given by [30]:

$$
F_{B}=-\frac{\rho}{4 \pi r_{12}^{2}}<\dot{V}_{1} \dot{V}_{2}>
$$

where $V_{1}$ and $V_{2}$ are the volume of bubble 1 and 2 respectively. $<>$ denotes the time average.

Besides Bjerknes forces, the bubbles experience drag and buoyancy forces from the sur- 
rounding liquid. The drag forces in the $\mathrm{x}\left(F_{v x}\right)$ and $\mathrm{y}\left(F_{v y}\right)$ axes are given by [37]

$$
\begin{gathered}
F_{v x}=-12 \pi \eta R_{n}\left(\dot{x}_{n}-v_{e}\right) \\
F_{v y}=-12 \pi \eta R_{n} \dot{y}_{n}
\end{gathered}
$$

where $\eta$ is the liquid viscosity. $x_{n}$ and $y_{n}$ are the positions of the $n$th bubble center on the $\mathrm{x}$ and $\mathrm{y}$ axes. $v_{e}$ is the liquid velocity that is generated by the imposed acoustic field at the center of the bubble

$$
v_{e}=\frac{P_{a}}{\rho c} \cos (\omega t) \cos \left(k d_{n}\right)
$$

where $c$ is the liquid velocity. Here a plane standing wave is assumed and boundary layers at walls are neglected.

The buoyancy force in the $\mathrm{y}$ axis is

$$
F_{\text {buoy }}=\frac{4 \pi}{3} R_{n}^{3}\left(\rho-\rho_{\text {gas }}\right)
$$

where $\rho_{\text {gas }}$ is the density of gas inside a bubble.

Therefore, the translational equations of the $n$th bubble in a multi-bubble environment are given by [38]

$$
\ddot{x}_{n}+\frac{3 \dot{R}_{n} \dot{x}_{n}}{R_{n}}=\frac{3 F_{e x}}{2 \pi \rho R_{n}^{3}}
$$




$$
\ddot{y}_{n}+\frac{3 \dot{R}_{n} \dot{y}_{n}}{R_{n}}=\frac{3 F_{e y}}{2 \pi \rho R_{n}^{3}}
$$

where $F_{e x}$ and $F_{e y}$ are the external forces in the $\mathrm{x}$ axis and $\mathrm{y}$ axis respectively. $F_{e x}$ represents the forces in the $\mathrm{x}$ axis which is equal to the sum of the primary Bjerknes force $\left(F_{p}\right)$, the viscous drag force $\left(F_{v x}\right)$ and the $\mathrm{x}$ axis component of the secondary Bjerknes forces $\left(F_{B} \cos \theta\right.$ in Fig. 2), which includes the forces generated by the boundaries and neighboring bubbles. $F_{e y}$ is the sum of buoyancy force $\left(F_{b}\right)$, viscous force $\left(F_{v y}\right)$ and the y axis component of the secondary Bjerknes forces $F_{B} \sin \theta$ (including the influences from the boundaries and neighboring bubbles in Fig. 2).

Furthermore, the time-varying bubble radius is calculated based on the Keller-Miksis equation [39]. In a multi-bubble environment, the Keller-Miksis equation needs to be expanded to include the influences from the boundary conditions and neighboring bubbles. By incorporating equation (2) into the Keller-Mikisis equation, the oscillation of the $n$th bubble is obtained:

$$
\begin{gathered}
\left(1-\frac{\dot{R}_{n}}{c}\right) R_{n} \ddot{R}_{n}+\left(\frac{3}{2}-\frac{\dot{R}_{n}}{2 c}\right) \dot{R}_{n}^{2}-\frac{1}{\rho}\left(1+\frac{\dot{R}_{n}}{c}\right) P_{s c}-\frac{R_{n}}{\rho c} \dot{P}_{s c}=\frac{\dot{x}_{n}^{2}}{4}-\sum_{\substack{m=1 \\
m \neq n}}^{N} \frac{1}{r_{n m}}\left(2 \dot{R}_{m}^{2} R_{m}+R_{m}^{2} \ddot{R}_{m}\right) \\
P_{s c}=\left(P_{0}+\frac{2 \sigma}{R_{n 0}}\right)\left(\frac{R_{n 0}}{R_{n}}\right)^{3 \gamma}-\frac{2 \sigma}{R_{n}}-\frac{4 \eta \dot{R}_{n}}{R_{n}}-P_{0}-P_{e x}
\end{gathered}
$$

where $R_{n 0}$ is the equilibrium radius of the $n$th bubble and an ensemble of $N$ bubbles is considered. $r_{n m}$ is the distance between the center of the $n$th and $m$ th bubbles. $P_{0}$ is the hydrostatic pressure, $\sigma$ is the surface tension, $\gamma$ is the polytropic exponent of the gas within the bubble. $P_{e x}$ is the external driving signal which is defined as a standing wave here: 


$$
P_{e x}=P_{a} \sin (\omega t) \sin \left(k d_{n}\right)
$$

The left terms of equation (10) are the modified Keller-Miksis equation for the $n$th bubble. This modified Keller-Miksis equation is coupled to the velocity of the $n$th bubble through the first term on the right, and to the pressure emitted or scattered by the neighboring bubbles through the second term on the right.

The resonance frequency of a bubble is [37]:

$$
f_{\text {res }}=\frac{1}{2 \pi R_{0}} \sqrt{\frac{3 \gamma P_{0}}{\rho}\left(1+\frac{2 \sigma}{P_{0} R_{0}}\right)-\frac{2 \sigma}{R_{0} \rho}}
$$

A total velocity is defined here as a function of time:

$$
v_{\text {total }}(t)=\sqrt{\dot{x}_{n}^{2}(t)+\dot{y}_{n}^{2}(t)}
$$

\section{Results}

In this section, the measured acoustic standing wave field and observed bubble translations within the resonator are shown. The values of the physical parameters used in this study are $f=46.8 \mathrm{kHz}, \rho=1000 \mathrm{~kg} / \mathrm{m}^{3}, P_{0}=101.3 \mathrm{kPa}, c=1480 \mathrm{~m} / \mathrm{s}, \sigma=0.072 \mathrm{~N} / \mathrm{m}, \gamma=1, \eta$ $=0.001 \mathrm{~Pa}^{*} \mathrm{~s}$. Experimental videos were recorded with a $3 \mathrm{~mm}$ by $1.8 \mathrm{~mm}$ viewing window at a frame rate of 100,000 frames/second.

\subsection{The acoustic standing wave field}

It has been shown in a previous work [34] that the one-dimensional equivalent electrical network of a transducer (1D model) is suitable for quantifying the pressure distribution of 
the present resonator. A simulated pressure distribution of the water layer with glass 1 at $46.8 \mathrm{kHz}$ is displayed in Fig. 4 for input signal amplitude of $4 \mathrm{~V}$ (peak). It can be seen from Fig. 4 that a calculated maximum pressure amplitude of $11.5 \mathrm{kPa}$ is located at the origin of the coordinate system $(x=0 \mathrm{~mm}$ in Fig. 1) and the pressure amplitude gradually drops to a minimum at the boundary between the water layer and glass $2(x=8 \mathrm{~mm})$ as indicated in Fig. 4. Also, the presence of glass 1 creates a pressure drop between its two sides (from $7.3 \mathrm{kPa}$ at $x=4$ to $6.9 \mathrm{kPa}$ at $x=4.1 \mathrm{~mm})$.

According to equation (13), the bubbles used in the tests (radii ranging from $10 \mu \mathrm{m}$ to $50 \mu \mathrm{m})$ are driven below their resonance frequencies. Therefore, the generated bubbles are expected to move in the direction from the bubble injection point at $x=5 \mathrm{~mm}$ towards the pressure anti-node at $x=0 \mathrm{~mm}$.

\subsection{Bubble translation in the acoustic standing wave field}

The translations of several bubbles moving from the bubble injection point towards glass 1 (Fig. 1) are displayed in Fig. 5 at a pressure amplitude of $11.5 \mathrm{kPa}$. The radius of bubble 1 in Fig. 5 is $42 \mu \mathrm{m}$, and the radii of the other bubbles are around $13 \mu \mathrm{m}$.

Initially, bubble 1 moves towards glass 1 at a faster speed than bubbles $2-14$. After $60 \mathrm{~ms}$, bubble 1 firstly arrives on glass 1 , while the following bubbles $2-14$ are moving on trajectories towards glass 1 and bubble 1 and start to form an arrow shape in the liquid medium. Bubble 3 and 4 are the first two to merge with bubble 1 at $400 \mathrm{~ms}$ followed by bubbles 5, 2, 6, and 7 sequentially. 


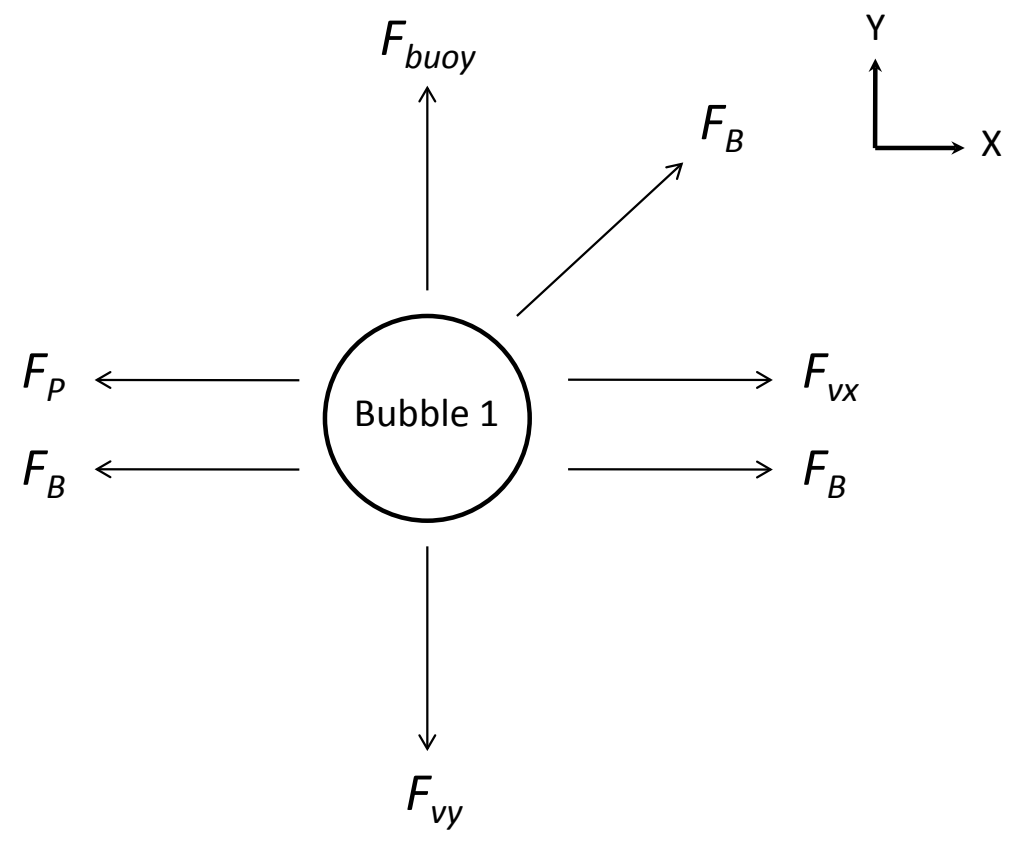

Figure 3: The relationship between different external forces on bubble 1

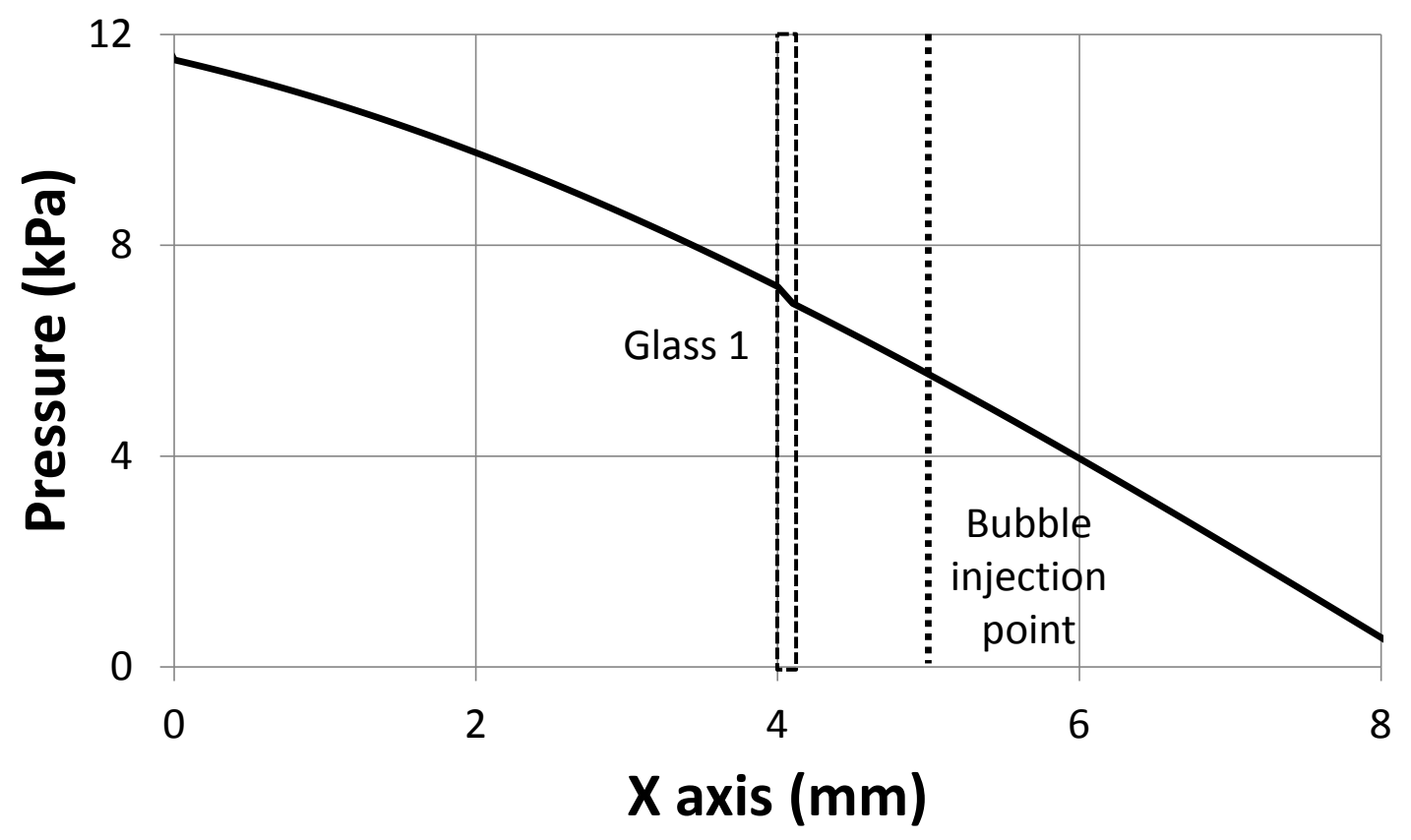

Figure 4: A simulated pressure distribution in the water layer with glass 1 at $46.8 \mathrm{kHz}$ for input amplitude of $4 \mathrm{~V}$. The position of glass 1 is indicated by the dashed square and the bubble injection point is shown by the dotted line. 

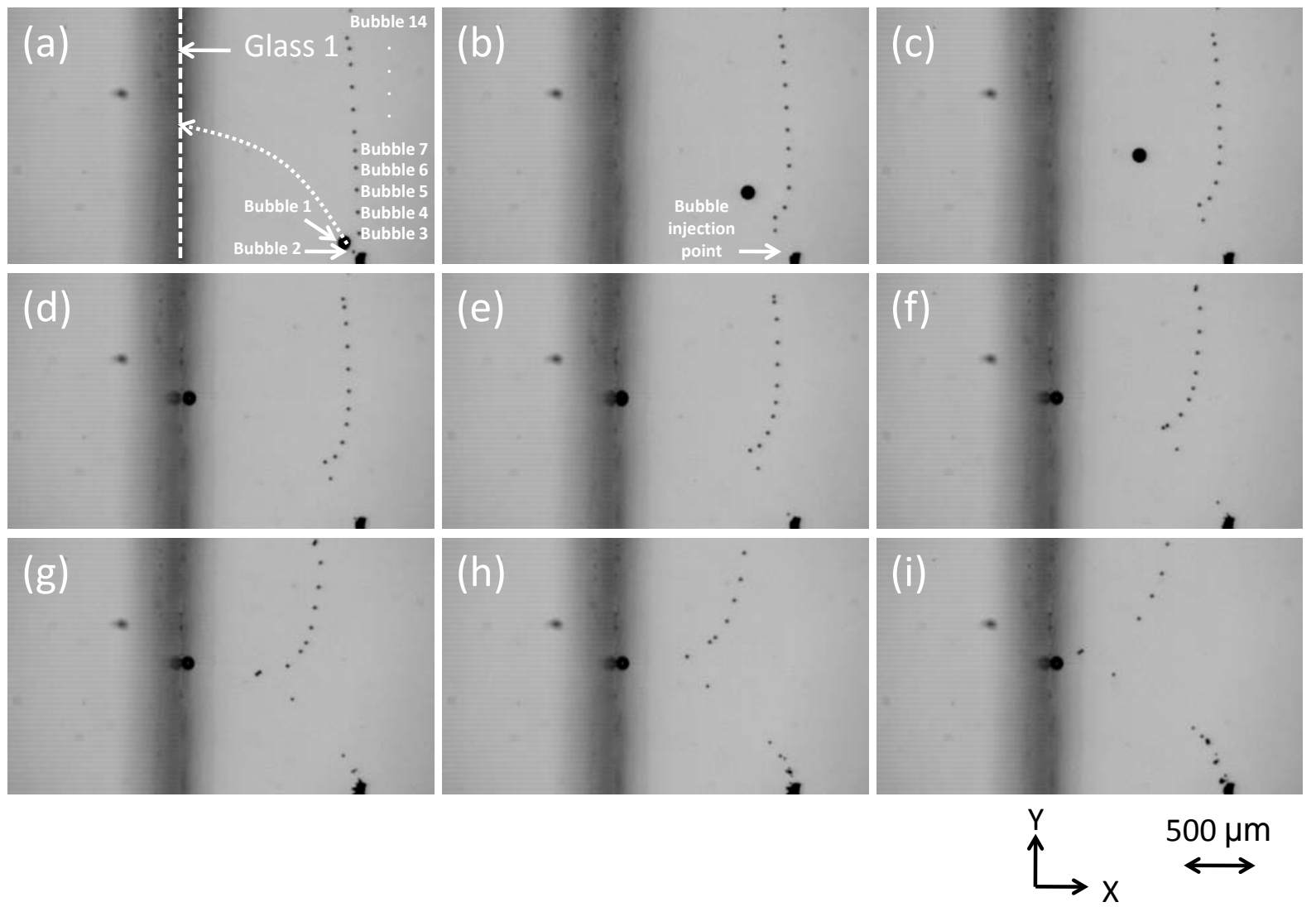

Figure 5: Selected frames from a video showing the translations of several bubbles from the injection point to glass 1 at (a) $0 \mathrm{~ms}$; (b) $20 \mathrm{~ms}$; (c) $40 \mathrm{~ms}$; (d) $60 \mathrm{~ms}$; (e) $100 \mathrm{~ms}$; (f) $200 \mathrm{~ms}$; (g) $300 \mathrm{~ms}$; (h) $400 \mathrm{~ms}$; (i) $500 \mathrm{~ms}$. The pressure amplitude was $11.5 \mathrm{kPa}$.

\section{Discussion}

The translation of a bubble in a liquid medium is the outcome of the competition of different external forces. The bubble motion is sensitive to the changes of surrounding environment, such as the presence of neighboring bubbles and boundary surfaces. In this section, the translations of bubbles are investigated by analyzing the relationship between acoustic and hydrodynamic forces exerted on the bubbles. All the bubble translations shown in Fig. 5 were studied and bubbles 1, 3, 5, and 7 are chosen here to illustrate the force relationship. The influence of bubble size and pressure amplitude on the bubble translation 
is also explored.

\subsection{The translation of bubble 1}

Recalling the force analysis in section 3, the translation of bubble 1 can be examined in the $\mathrm{x}$ and $\mathrm{y}$ axes respectively and the relationship of several main external forces in the $\mathrm{x}$ axis is shown in Fig. 6 (e).

In the $\mathrm{x}$ axis, after the ultrasound is switched on, bubble 1 is mainly controlled by the primary Bjerknes force and starts to move in the direction towards glass 1 from the bubble injection point. It can be seen from Fig. 6 (b) that the velocity of bubble 1 in the experiment suddenly rises from 0 to $16 \mathrm{~mm} / \mathrm{s}$ and then maintains the speed until arriving at $x=4.4 \mathrm{~mm}$. After that, the secondary Bjerknes force from glass 1 grows stronger and starts to outweigh the primary Bjerknes force. The secondary Bjerkens force from glass 2 and a nearby bubble 2 can be neglected at this stage. The velocity of bubble 1 surges up from $20 \mathrm{~mm} / \mathrm{s}$ at $x=4.4$

$\mathrm{mm}$ to $207 \mathrm{~mm} / \mathrm{s}$ at $x=4.07 \mathrm{~mm}$. Good agreement is found between the experiment and theoretical prediction of the bubble $1 \mathrm{x}$ axis velocity.

In the $y$ axis, it is anticipated that the buoyancy force is stronger than the drag force at the beginning, but later on a balance is reached between the two forces. From theory, bubble 1 is expected to move at a steady speed of $15 \mathrm{~mm} / \mathrm{s}$ after taking off from the bubble injection point. However, in the experiment, the velocity of bubble 1 witnesses a rise from 0 $\mathrm{mm} / \mathrm{s}$ at $y=0.16 \mathrm{~mm}$ to $20 \mathrm{~mm} / \mathrm{s}$ at $y=0.18 \mathrm{~mm}$ followed by a gradual drop to $6 \mathrm{~mm} / \mathrm{s}$ at $y=0.89 \mathrm{~mm}$. From the point of view of force, it is possible that the primary Bjerknes force on bubble 1 is weaker than expected since the standing wave field has not been fully established at the moment when the sound field is switched on. In the later phase, especially 
when bubble 1 is moving close to glass 1, the full strength primary Bjerknes force and the attractive force from glass 1 greatly accelerate the bubble motion in the $\mathrm{x}$ axis which in turn shortens the traveling distance in the y axis over the same period. The velocity in the $\mathrm{y}$ axis, therefore, is decreasing when bubble 1 is approaching glass 1 . This effect can also be seen in the time lag of the traveling time between theory and experiment (Fig. $6(\mathrm{~d}))$. In the experiment, the time for bubble 1 to move from the bubble injection point to glass 1 is 60 ms which is longer than the $52 \mathrm{~ms}$ from the theory due to the insufficient primary Bjerknes force experienced by bubble 1 at the beginning.

\subsection{The translations of bubbles 3,5 , and 7}

It can be seen from Fig. 5 that bubbles $2-14$ move at a much slower speed than bubble 1 and their translations behave in a different manner. To explain such behavior, bubbles 3,5 , and 7 are chosen here as the example bubbles since they represent the typical translational behavior experienced by all other bubbles.

By decomposing the external forces into the $\mathrm{x}$ axis force and $\mathrm{y}$ axis force, one can study the bubble translation using the same procedure for bubble 1. Initially, the motion of bubble 3 in the $\mathrm{x}$ axis, for example, is mainly controlled by the secondary Bjerknes force from bubble 1. When bubble 1 and 3 are still close to each other, this secondary Bjerknes force is stronger than the primary Bjerknes force and results in a surge in velocity in the $\mathrm{x}$ axis (Fig. 7 (b)). As bubble 1 is moving at a faster speed towards glass 1 , the distance between bubble 3 and 1 grows to the extent that such bubble-bubble interaction is weaker than the primary Bjerknes force. The predicted velocity in the $\mathrm{x}$ axis, therefore, decreases from $20 \mathrm{~mm} / \mathrm{s}$ at $x=5.25 \mathrm{~mm}$ to $4 \mathrm{~mm} / \mathrm{s}$ at $x=5 \mathrm{~mm}$, while in the experiment the change of velocity over the same period 

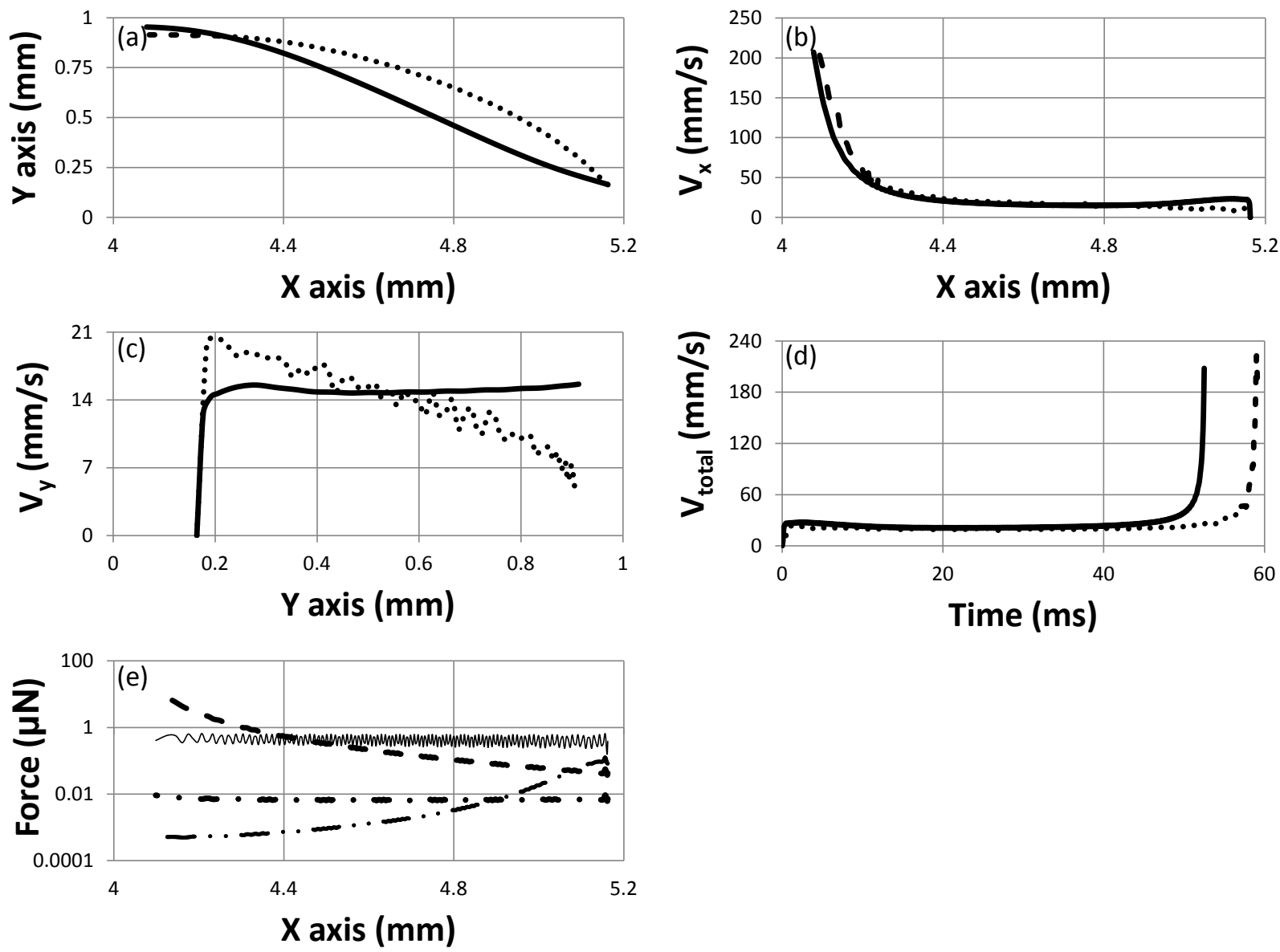

Figure 6: The translation of bubble 1 at $46.8 \mathrm{kHz}$ (a) bubble trajectory, experimental result $\cdots$, theoretical prediction -; (b) velocity in the $\mathrm{x}$ axis, experimental result $\cdots$, theoretical prediction - ; (c) velocity in the $\mathrm{y}$ axis, experimental result $\cdots$, theoretical prediction -; (d) total velocity, experimental result $\cdots$, theoretical prediction -; (e) relationship between different forces, primary Bjerknes force - , secondary Bjerknes force from glass $1--$, secondary Bjerknes force from glass $2-\cdots-$, secondary Bjerknes force from bubble $2-\cdot-$. The pressure amplitude was $11.5 \mathrm{kPa}$. 
is smaller than the expectation but is still noticeable. From Fig. 7 (a), it can be seen that the predicted trajectory of bubble 3 between $x=5.25 \mathrm{~mm}$ to $x=5 \mathrm{~mm}$ also deviates from the experimental result. As discussed in the bubble 1 case, at the beginning of the experiment, the standing wave field in the experiment is weaker than the theoretical prediction which forces bubble 1 to move away from the anticipated trajectory. The trajectory of bubble 3 is consequently changed since the secondary Bjerknes force between bubble 1 and 3 dominates the translation of bubble 3 over that period. However, the shape of the velocity profile between $x=5.25 \mathrm{~mm}$ and $x=5 \mathrm{~mm}$ in the experiment is still consistent with that of the theory.

After the arrival of bubble 1 on to glass 1, the primary Bjerknes force and the secondary Bjerknes force from bubble 1 become the major factors that control the translation of bubble 3. The velocity of bubble 3 in the $\mathrm{x}$ axis between $x=5 \mathrm{~mm}$ and $x=4.5 \mathrm{~mm}$ is around 3 $\mathrm{mm} / \mathrm{s}$. When the distance between bubble 3 and bubble 1 decreases, the secondary Bjerknes force from bubble 1 is again dominating the motion of bubble 3 in the $\mathrm{x}$ axis. Moreover, within the near field of bubble 1, the secondary Bjerknes force from glass 1 becomes stronger than the primary Bjerknes force and contributes to the boost of velocity along with the interaction force between bubble 1 and 3 . The velocity of bubble 3 jumps from $3 \mathrm{~mm} / \mathrm{s}$ at $x=4.5 \mathrm{~mm} / \mathrm{s}$ to $90 \mathrm{~mm} / \mathrm{s}$ at $x=4.18 \mathrm{~mm}$, which is close to the predicted $112 \mathrm{~mm} / \mathrm{s}$.

In the y axis, initially, bubble 3 is lifted by the attractive force from bubble 1 and the buoyancy force. As bubble 1 is moving away at a faster speed, the bubble 1 and 3 interaction diminishes as a function of time and the bubble 3 y axis velocity remains at $3 \mathrm{~mm} / \mathrm{s}$ between $y=0.17 \mathrm{~mm}$ and $y=0.7 \mathrm{~mm}$ in Fig. 7 (c). After that, the attractive force from bubble 1 significantly accelerates the velocity when bubble 3 approaches bubble 1 . A $94 \mathrm{~mm} / \mathrm{s}$ 
velocity is seen in the theoretical prediction at $y=0.88 \mathrm{~mm}$ which is higher than the 27 $\mathrm{mm} / \mathrm{s}$ one observed in the experiment. Since bubble 3 moves on a trajectory which is not perfectly matching the theoretical prediction, the consequent bubble translation, especially at the moment when bubble 1 and 3 are close enough, could be different from what is expected from theory. Therefore, the y axis velocity in the experiment is different from that of the simulation. The predicted overall traveling time for bubble 3 to move from the bubble injection point to glass 1 is in quantitative agreement with the experimental result as shown in Fig. 7 (d).

A similar analysis was also applied to bubble 5 and 7 in Fig. 8 and Fig. 9 respectively. A surge of $\mathrm{x}$ axis velocity due to the increase of secondary Bjerknes force from the surface is seen for both bubbles in Fig. 8 (b) and Fig. 9 (b). The observed maximum x axis velocity of bubbles 5 and 7 are $109 \mathrm{~mm} / \mathrm{s}$ and $214 \mathrm{~mm} / \mathrm{s}$, which are lower than the anticipated 200 $\mathrm{mm} / \mathrm{s}$ and $300 \mathrm{~mm} / \mathrm{s}$ from the model. Ideally, the detection of velocity change, especially at the moment when the bubble is approaching the boundary, requires a high frame rate. However, the limited frame rate used in the experiment was unable to provide the small time interval to construct the accurate velocity information at the final moment when the bubble contacting the surface and therefore results in a lower than expected $\mathrm{x}$ axis velocity in Fig. 8 (b) and Fig. 9 (b).

It needs to be pointed out here that the influence from bubble 1 on the nearby bubbles decreases with an increase of distance between the bubbles. For the bubbles in the far field of bubble 1, a weaker attractive force generated from bubble 1 was anticipated. It can be seen from Fig. 9 that since bubble 7 moves at a slow speed, the standing wave field has sufficient time to be established in the $\mathrm{x}$ axis. The predicted translation of bubble 7 , therefore, is in 
good agreement with the experimental result. On the other hand, there is a discrepancy of bubble trajectory between the experiment and the prediction for bubble 5 in Fig. 8 (a) which is the consequence of the deviation between the observed and calculated trajectory of bubble 1 shown in Fig. 6 (a).

\subsection{Parametric study}

To transport a large amount of bubbles of given size to an appointed position on a surface, one needs to optimize the external forces exerted on the bubbles, such as primary and secondary Bjerknes forces. In section 3, it is seen that the Bjerknes forces are directly related to the bubble size and external pressure amplitude. In this section, the influence of different bubble sizes and pressure amplitudes on the bubble translation is discussed.

The translation of the $13 \mu \mathrm{m}$ bubbles are sensitive to the changes of acoustic and hydrodynamic forces. Let us assume a bubble 1 of radius of $42 \mu \mathrm{m}$ is fixed on glass 1 at $x=4 \mathrm{~mm}$, $y=9 \mathrm{~mm}$, and another bubble 2 can move freely in the water layer. The driving frequency is kept at $46.8 \mathrm{kHz}$. The calculated forces in Fig. 10 (a2, b2, c2) are represented by their absolute values.

Firstly, three radii of bubble 2, 6.5 $\mu \mathrm{m}, 13 \mu \mathrm{m}$, and $26 \mu \mathrm{m}$, are used in Fig. 10 (a1, a2) at $11.5 \mathrm{kPa}$. Fig. 10 (a1) shows that changing the radius of bubble 2 can hardly alter its trajectory. The secondary Bjerknes force between bubble 1 and 2 is proportional to their sizes and therefore an increase of the size of bubble 2 results in an increase of secondary Bjerknes force as well, which in turn accelerates the velocity of bubble 2 . The traveling time of bubble 2 was found from our calculations to be shortened from $2500 \mathrm{~ms}$ for the $6.5 \mu \mathrm{m}$ bubble to $100 \mathrm{~ms}$ for the $26 \mu \mathrm{m}$ one. 

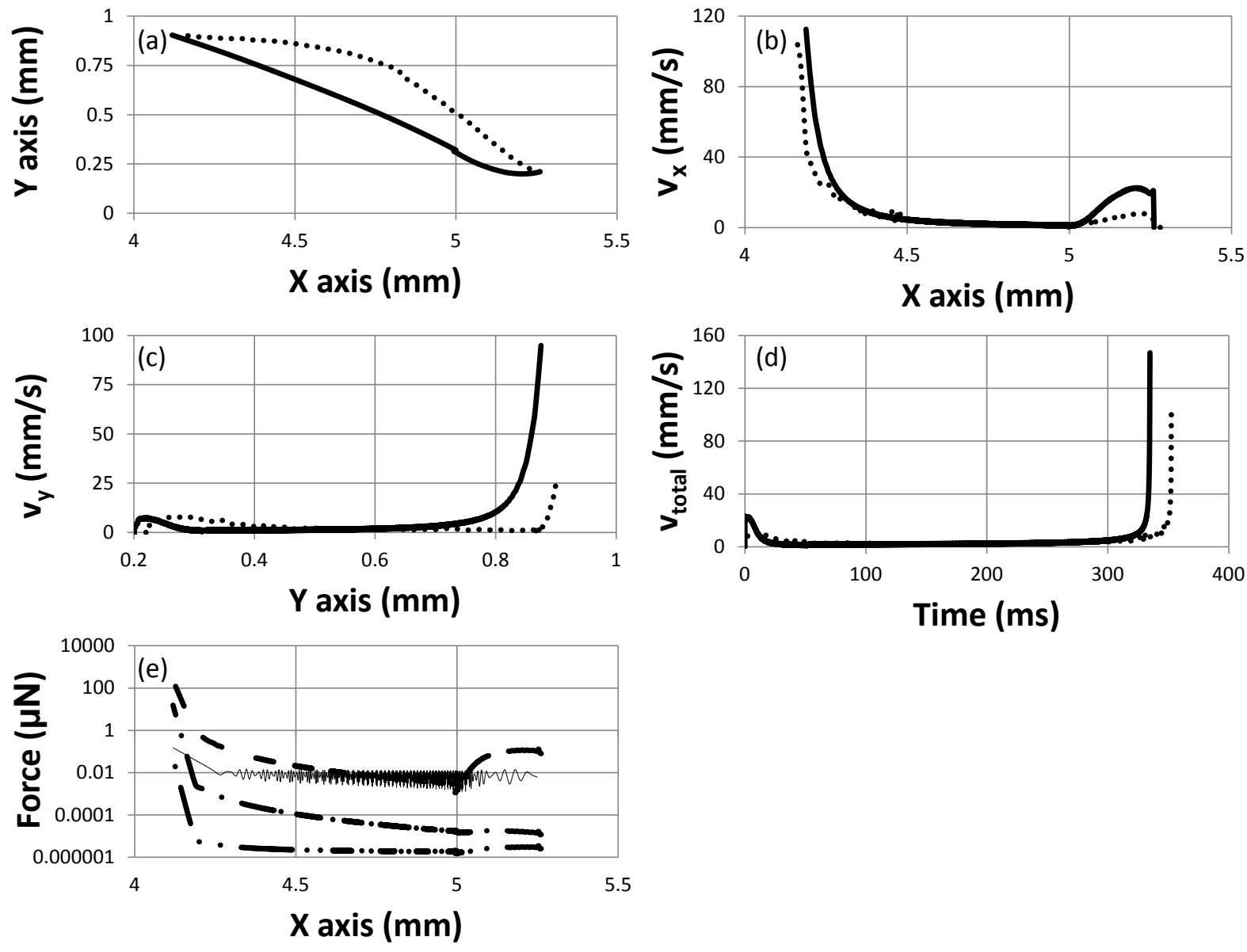

Figure 7: The translation of bubble 3 at $46.8 \mathrm{kHz}$ (a) bubble trajectory, experimental result $\cdots$, theoretical prediction -; (b) velocity in the $\mathrm{x}$ axis, experimental result $\cdots$, theoretical prediction - ; (c) velocity in the $\mathrm{y}$ axis, experimental result $\cdots$, theoretical prediction -; (d) total velocity, experimental result $\cdots$, theoretical prediction -; (e) relationship between different forces, primary Bjerknes force - , secondary Bjerknes force from glass $1--$, secondary Bjerknes force from glass $2-\cdots-$, secondary Bjerknes force from bubble $2-\cdot-$. The pressure amplitude was $11.5 \mathrm{kPa}$. 

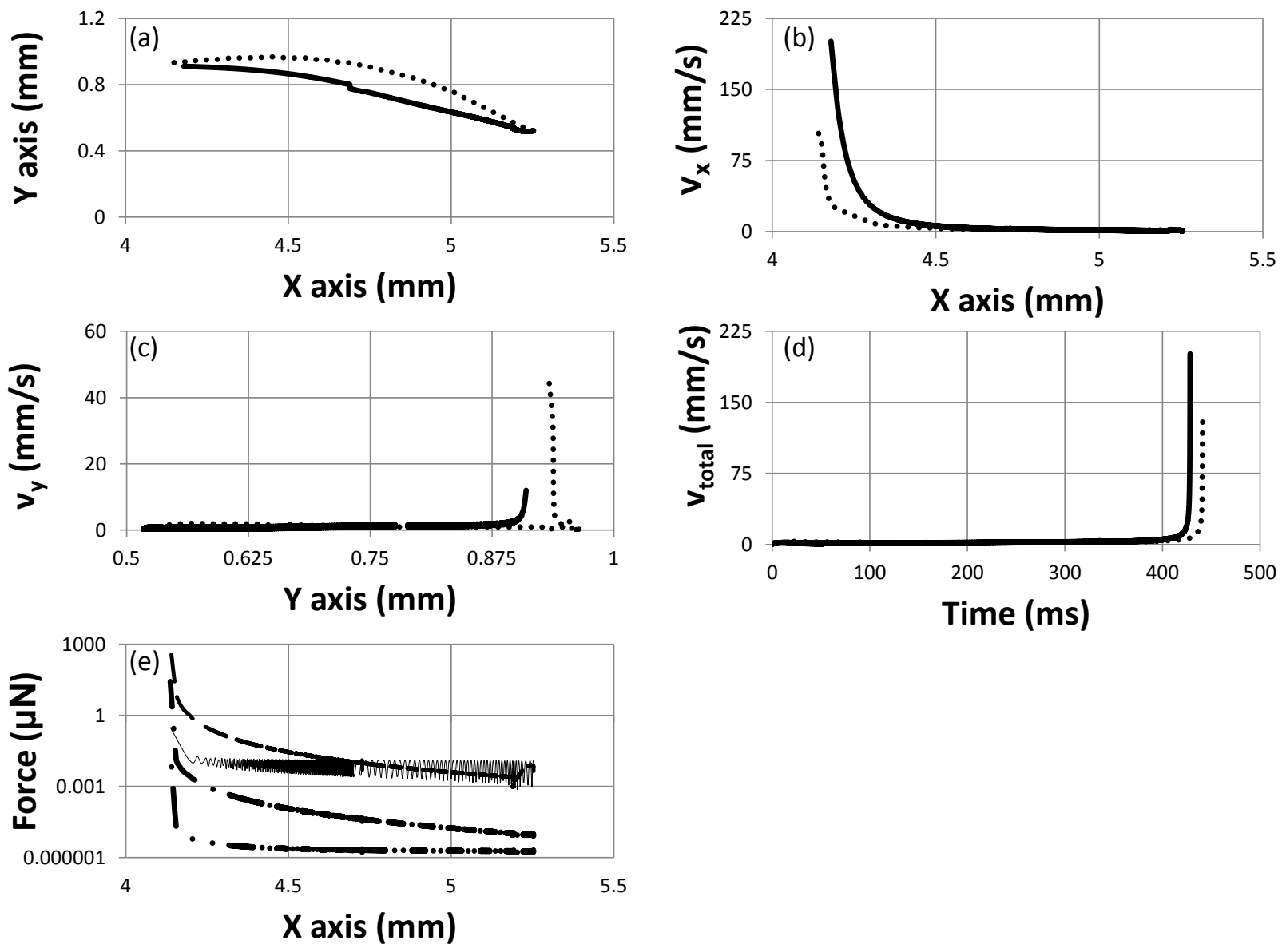

Figure 8: The translation of bubble 5 at $46.8 \mathrm{kHz}$ (a) bubble trajectory, experimental result $\cdots$, theoretical prediction -; (b) velocity in the $\mathrm{x}$ axis, experimental result $\cdots$, theoretical prediction - ; (c) velocity in the $\mathrm{y}$ axis, experimental result $\cdots$, theoretical prediction -; (d) total velocity, experimental result $\cdots$, theoretical prediction -; (e) relationship between different forces, primary Bjerknes force -, secondary Bjerknes force from glass $1--$, secondary Bjerknes force from glass $2-\cdots-$, secondary Bjerknes force from bubble $2-\cdot-$. The pressure amplitude was $11.5 \mathrm{kPa}$. 

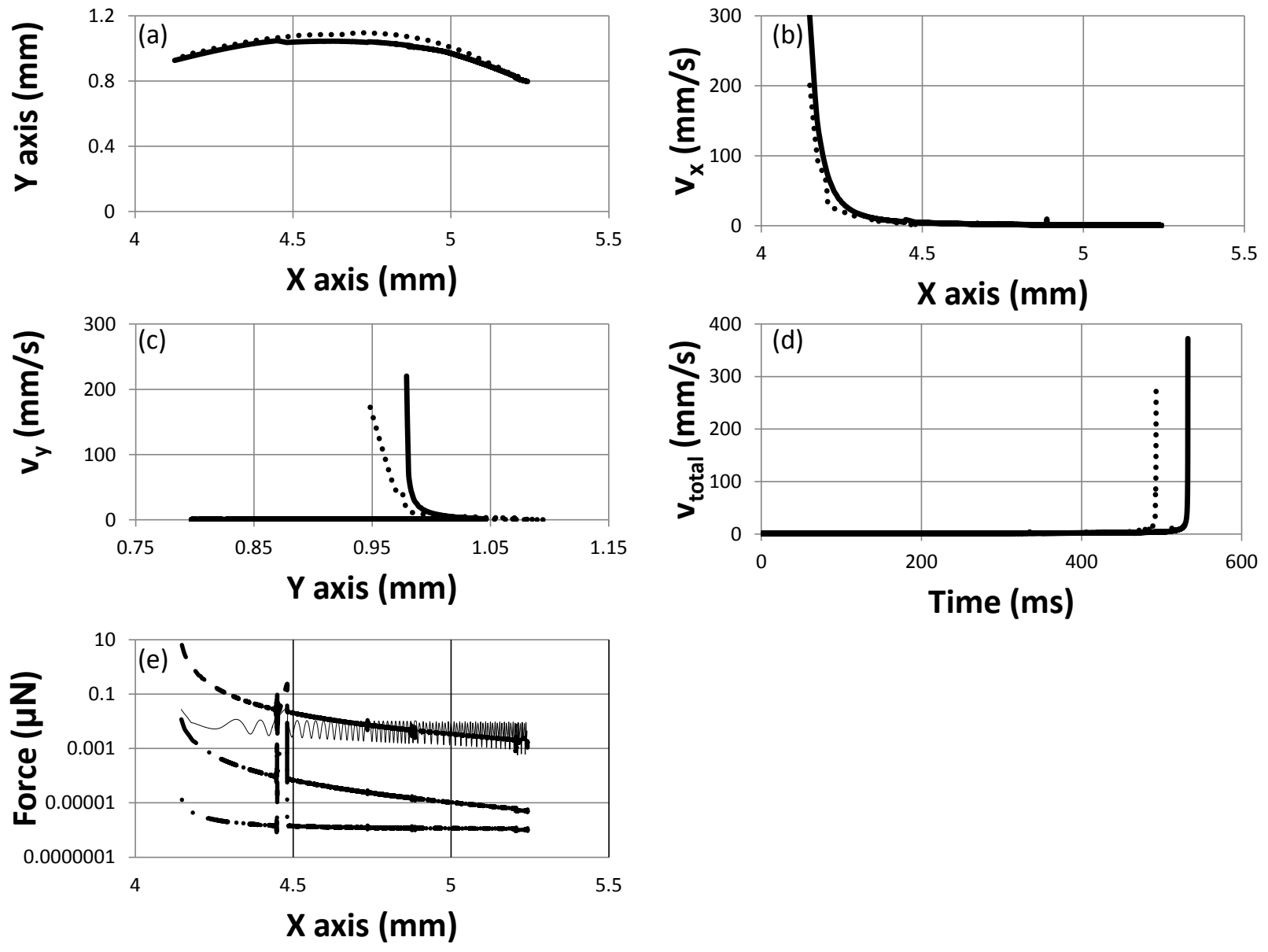

Figure 9: The translation of bubble 7 at $46.8 \mathrm{kHz}$ (a) bubble trajectory, experimental result $\cdots$, theoretical prediction -; (b) velocity in the $\mathrm{x}$ axis, experimental result $\cdots$, theoretical prediction - ; (c) velocity in the $\mathrm{y}$ axis, experimental result $\cdots$, theoretical prediction -; (d) total velocity, experimental result $\cdots$, theoretical prediction -; (e) relationship between different forces, primary Bjerknes force - , secondary Bjerknes force from glass $1--$, secondary Bjerknes force from glass $2-\cdots-$, secondary Bjerknes force from bubble $2-\cdot-$. The pressure amplitude was $11.5 \mathrm{kPa}$. 
Secondly, the radius of bubble 2 is assumed to be $13 \mu \mathrm{m}$, and the radius of bubble 1 is varying from $25 \mu \mathrm{m}$ to $100 \mu \mathrm{m}$. The pressure amplitude is $11.5 \mathrm{kPa}$. A striking difference of bubble 2 trajectory is noticed in Fig. 10 (b1). Bubble 2 experiences much less secondary Bjerknes force from the $25 \mu \mathrm{m}$ bubble 1 than from the $100 \mu \mathrm{m}$ one. The $100 \mu \mathrm{m}$ bubble exerts a repulsive instead of attractive force on bubble 2. It is well-known that the secondary Bjerknes force between two bubbles can shift from an attractive force when the bubbles are oscillating in phase, to a repulsive force when their oscillations are out of phase [27, 37]. Based on equation (13), at $46.8 \mathrm{kHz}$, the $100 \mu \mathrm{m}$ bubble is driven above its resonance frequency, while bubble 2 is smaller than the resonance size. Therefore, the secondary Bjerknes force between these two bubbles shifts from an attractive one to a repulsive one in the $100 \mu \mathrm{m}$ (bubble 1) case.

Thirdly, the radii of bubble 1 and 2 are kept as $42 \mu \mathrm{m}$ and $13 \mu \mathrm{m}$ respectively. The pressure amplitude is increased from $5.25 \mathrm{kPa}$ to $23 \mathrm{kPa}$ (Fig. 10 (c1, c2)). At a lower

pressure amplitude, bubble 2 experiences a smaller secondary Bjerknes force from bubble 1 which only starts to divert the trajectory of bubble 2 within the near field (Fig. 10 (c1)). At a higher pressure amplitude, the bubble 2 migrates directly towards bubble 1 at a faster speed due to the increase of interaction between the bubbles (Fig. 10 (c2)).

\section{Conclusion}

The collective bubble dynamics near a surface in a weak acoustic standing wave field is shown. The bubble translation in a multi-bubble environment was achieved by using a multilayered resonator which created an uniform one-dimensional acoustic standing wave field in a water layer. The bubble motion was modeled by a pair of modified Keller-Miksis equation 

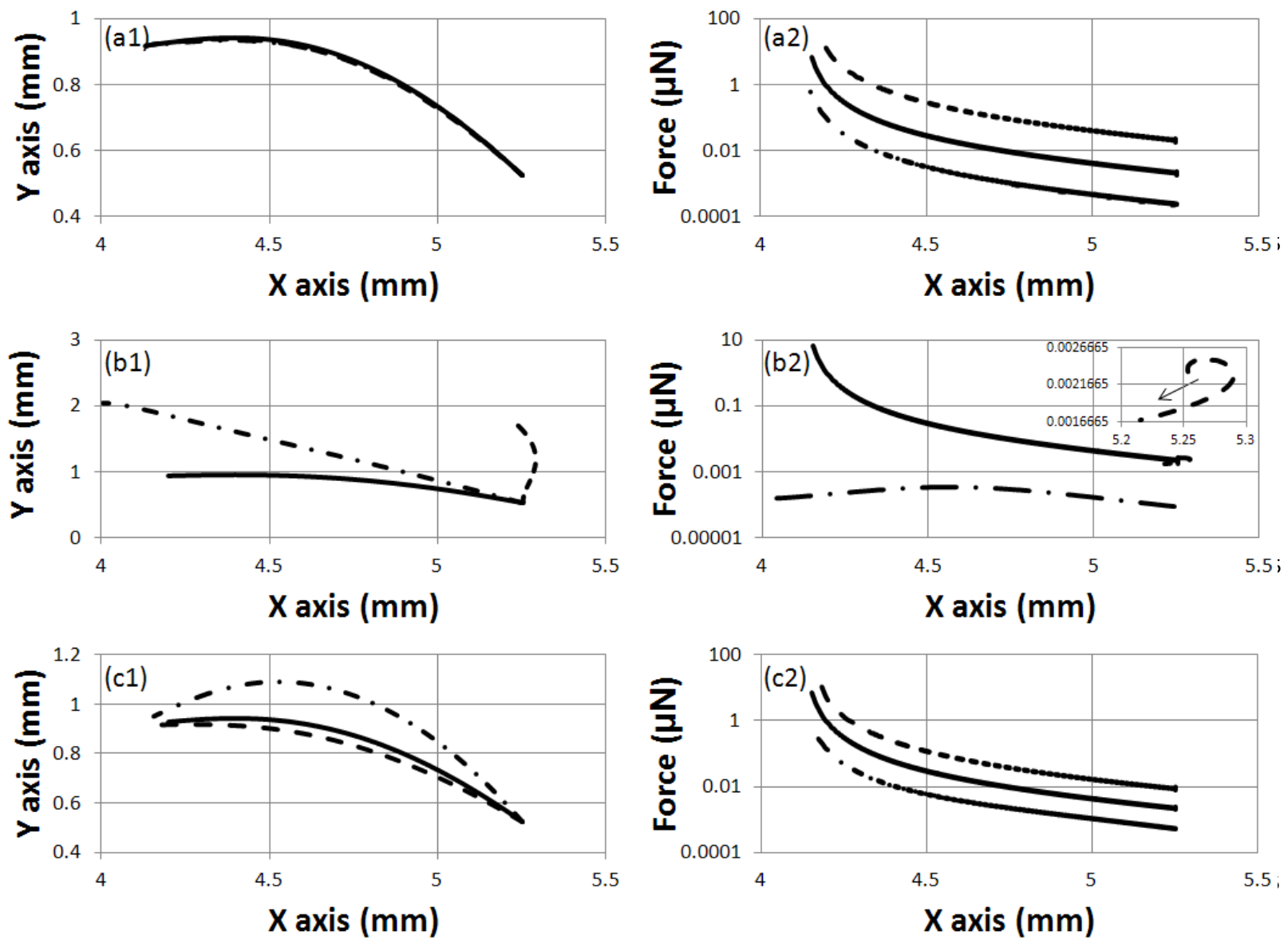

Figure 10: A parametric study of the bubble translation under different conditions. The calculated forces are represented by their absolute values. (a1) at $11.5 \mathrm{kPa}$, the radii of bubble 2 are $6.5 \mu \mathrm{m}(-\cdot-), 13 \mu \mathrm{m}(-)$, and $26 \mu \mathrm{m}(--)$; (a2) at $11.5 \mathrm{kPa}$, the secondary Bjerknes force on bubble 2 with radii of $6.5 \mu \mathrm{m}(-\cdot-), 13$ $\mu \mathrm{m}(-)$, and $26 \mu \mathrm{m}(--)$; (b1) at $11.5 \mathrm{kPa}$, the radii of bubble 1 are $25 \mu \mathrm{m}(-\cdot-), 50 \mu \mathrm{m}(-)$, and $100 \mu \mathrm{m}$ $(--)$; (b2) at $11.5 \mathrm{kPa}$, the secondary Bjerknes force on bubble 2 with bubble 1 of radii of $25 \mu \mathrm{m}(-\cdot-)$, $50 \mu \mathrm{m}(-)$, and $100 \mu \mathrm{m}(--)$. The secondary force between the $100 \mu \mathrm{m}$ bubble 1 and $13 \mu \mathrm{m}$ bubble 2 is shown in the inset; (c1) for a pair of bubbles of radii of $50 \mu \mathrm{m}$ and $13 \mu \mathrm{m}$, the pressure amplitude is 5.25 $\mathrm{kPa}(-\cdot-), 11.5 \mathrm{kPa}(-)$, and $23 \mathrm{kPa}(--) ;(\mathrm{c} 2)$ the secondary Bjerknes force between the bubbles at 5.25 $\mathrm{kPa}(-\cdot-), 11.5 \mathrm{kPa}(-)$, and $23 \mathrm{kPa}(--)$; 
and bubble translation equation. The influence of several acoustic and hydrodynamic forces on the bubble translation was investigated. It was found that the bubble translation near a surface in a multi-bubble environment was mainly controlled by the primary Bjerknes force imposed by the acoustic field, secondary Bjerknes forces introduced by a surface and neighboring bubbles, and buoyancy force from the surrounding liquid. The primary Bjerknes force dominated the bubble translation when the bubble was far away from the surface and was outweighed by the secondary Bjerknes force from the boundary when the bubble was approaching the surface. Moreover, a strong secondary Bjerknes force generated by a neighboring bubble was noticed in the experiment. The bubble-bubble interaction forced nearby bubbles to move on trajectories towards the target bubble instead of the positions that they would have moved to in the absence of the target bubble. It was also seen from a parametric study that increasing the pressure amplitude can enhance the interaction between two bubbles and force bubbles to move at a faster speed. The secondary Bjerknes force between two bubbles can shift from an attractive one when two bubbles oscillate in phase to a repulsive one when their oscillations are out of phase. All of these effects can be decided quantitatively with the presented theory.

\section{Acknowledgement}

X.X and F.C would like to acknowledge financial support from Lam Research AG (SEZStrasse 1, 9050 Villach, Austria). R.M gratefully acknowledges financial support by the Austrian Federal Ministry of Economy, Family and Youth and the Austrian National Foundation for Research, Technology and Development. 


\section{References}

[1] A. Vogel, W. Lauterborn, and R. Timm. Optical and acoustic investigations of the dynamics of laserproduced cavitation bubbles near a solid boundary. Journal of Fluid Mechanics, 206(-1):299-338, 1989.

[2] J.R. Blake and DC Gibson. Cavitation bubbles near boundaries. Annual Review of Fluid Mechanics, 19(1):99-123, 1987.

[3] O. Lindau and W. Lauterborn. Cinematographic observation of the collapse and rebound of a laserproduced cavitation bubble near a wall. Journal of Fluid Mechanics, 479(-1):327-348, 2003.

[4] P. Prentice, A. Cuschieri, K. Dholakia, M. Prausnitz, and P. Campbell. Membrane disruption by optically controlled microbubble cavitation. Nature Physics, 1(2):107-110, 2005.

[5] A. Philipp and W. Lauterborn. Cavitation erosion by single laser-produced bubbles. Journal of Fluid Mechanics, 361(-1):75-116, 1998.

[6] C.D. Ohl, M. Arora, R. Dijkink, V. Janve, and D. Lohse. Surface cleaning from laser-induced cavitation bubbles. Applied Physics Letters, 89(7):074102, 2009.

[7] W. Kim, T.H. Kim, J. Choi, and H.Y. Kim. Mechanism of particle removal by megasonic waves. Applied Physics Letters, 94(8):081908, 2009.

[8] WD Song, MH Hong, B. Lukyanchuk, and TC Chong. Laser-induced cavitation bubbles for cleaning of solid surfaces. Journal of applied physics, 95:2952, 2004.

[9] M.S. Plesset and R.B. Chapman. Collapse of an initially spherical vapour cavity in the neighbourhood of a solid boundary. Journal of Fluid Mechanics, 47(02):283-290, 1971.

[10] K. Sato, Y. Tomita, and A. Shima. Numerical analysis of a gas bubble near a rigid boundary in an oscillatory pressure field. The Journal of the Acoustical Society of America, 95:2416, 1994.

[11] J.R. Blake, G.S. Keen, R.P. Tong, and M. Wilson. Acoustic cavitation: the fluid dynamics of nonspherical bubbles. Philosophical Transactions of the Royal Society of London. Series A: Mathematical, Physical and Engineering Sciences, 357(1751):251, 1999.

[12] W. Lauterborn and T. Kurz. Physics of bubble oscillations. Reports on Progress in Physics, 73:106501, 2010.

[13] X. Xi, F. Yang, D. Chen, Y. Luo, D. Zhang, N. Gu, and J. Wu. A targeting drug-delivery model via interactions among cells and liposomes under ultrasonic excitation. Physics in Medicine and Biology, 53:3251, 2008.

[14] P. Marmottant and S. Hilgenfeldt. Controlled vesicle deformation and lysis by single oscillating bubbles. Nature, 423(6936):153-156, 2003.

[15] P. Marmottant, S. van der Meer, M. Emmer, M. Versluis, N. de Jong, S. Hilgenfeldt, and D. Lohse. A model for large amplitude oscillations of coated bubbles accounting for buckling and rupture. The Journal of the Acoustical Society of America, 118:3499, 2005.

[16] P. Marmottant, M. Versluis, N. de Jong, S. Hilgenfeldt, and D. Lohse. High-speed imaging of an ultrasound-driven bubble in contact with a wall:narcissus effect and resolved acoustic streaming. Experiments in fluids, 41(2):147-153, 2006.

[17] V. Garbin, D. Cojoc, E. Ferrari, E. Di Fabrizio, MLJ Overvelde, SM van Der Meer, N. de Jong, D. Lohse, and M. Versluis. Changes in microbubble dynamics near a boundary revealed by combined optical micromanipulation and high-speed imaging. Applied physics letters, 90:114103, 2007. 
[18] HJ Vos, B. Dollet, JG Bosch, M. Versluis, and N. de Jong. Nonspherical vibrations of microbubbles in contact with a wall-a pilot study at low mechanical index. Ultrasound in medicine and biology, $34(4): 685-688,2008$.

[19] H.J. Vos, B. Dollet, M. Versluis, and N. de Jong. Nonspherical shape oscillations of coated microbubbles in contact with a wall. Ultrasound in Medicine and Biology, 37(6):935-948, 2011.

[20] A.A. Doinikov, J.F. Haac, and P.A. Dayton. Modeling of nonlinear viscous stress in encapsulating shells of lipid-coated contrast agent microbubbles. Ultrasonics, 49(2):269-275, 2009.

[21] A.A. Doinikov and A. Bouakaz. Theoretical investigation of shear stress generated by a contrast microbubble on the cell membrane as a mechanism for sonoporation. The Journal of the Acoustical Society of America, 128:11, 2010.

[22] A.A. Doinikov and A. Bouakaz. Acoustic microstreaming around a gas bubble. The Journal of the Acoustical Society of America, 127:703, 2010.

[23] L.V. King. On the acoustic radiation pressure on spheres. Proceedings of the Royal Society of London. Series A, Mathematical and Physical Sciences, 147(861):212-240, 1934.

[24] A. Eller. Force on a bubble in a standing acoustic wave. The Journal of the Acoustical Society of America, 43:170, 1968.

[25] L.A. Crum. Bjerknes forces on bubbles in a stationary sound field. The Journal of the Acoustical Society of America, 57:1363, 1975.

[26] J. Rensen, D. Bosman, J. Magnaudet, C.D. Ohl, A. Prosperetti, R. Togel, M. Versluis, and D. Lohse. Spiraling bubbles: How acoustic and hydrodynamic forces compete. Physical Review Letters, 86(21):48194822, 2001.

[27] I. Akhatov, R. Mettin, CD Ohl, U. Parlitz, and W. Lauterborn. Bjerknes force threshold for stable single bubble sonoluminescence. Physical review E, 55(3):3747-3750, 1997.

[28] T. Barbat, N. Ashgriz, and C.S. Liu. Dynamics of two interacting bubbles in an acoustic field. Journal of Fluid Mechanics, 389(-1):137-168, 1999.

[29] AA Doinikov and ST Zavtrak. On the mutual interaction of two gas bubbles in a sound field. Physics of Fluids, 7:1923, 1995.

[30] R. Mettin, I. Akhatov, U. Parlitz, CD Ohl, and W. Lauterborn. Bjerknes forces between small cavitation bubbles in a strong acoustic field. Physical review E, 56(3):2924, 1997.

[31] R. Mettin, S. Luther, CD Ohl, and W. Lauterborn. Acoustic cavitation structures and simulations by a particle model d. Ultrasonics Sonochemistry, 6(1):25-30, 1999.

[32] U. Parlitz, R. Mettin, S. Luther, I. Akhatov, M. Voss, and W. Lauterborn. Spatio-temporal dynamics of acoustic cavitation bubble clouds. Philosophical Transactions of the Royal Society of London. Series A: Mathematical, Physical and Engineering Sciences, 357(1751):313, 1999.

[33] A.A. Doinikov. Mathematical model for collective bubble dynamics in strong ultrasound fields. The Journal of the Acoustical Society of America, 116:821, 2004.

[34] X. Xi, F. Cegla, M. Lowe, A. Thiemann, T. Nowak, R. Mettin, F. Holsteyns, and A. Lippert. Study on the bubble transport mechanism in an acoustic standing wave field. Ultrasonics, 51(8):1014-1025, 2011.

[35] PD Wilcox, RSC Monkhouse, P. Cawley, MJS Lowe, and BA Auld. Development of a computer model for an ultrasonic polymer film transducer system. NDT and E International, 31(1):51-64, 1998. 
[36] M. Hill, Y. Shen, and J.J. Hawkes. Modelling of layered resonators for ultrasonic separation. Ultrasonics, 40(1-8):385-392, 2002.

[37] TG Leighton. The acoustic bubble. Academic Press, 1997.

[38] A.A. Doinikov. Translational motion of a spherical bubble in an acoustic standing wave of high intensity. Physics of Fluids, 14:1420, 2002.

[39] J.B. Keller and M. Miksis. Bubble oscillations of large amplitude. The Journal of the Acoustical Society of America, 68(2):628-633, 1980. 